\title{
Technical Note: Regularization performances with the error consistency method in the case of retrieved atmospheric profiles
}

\author{
S. Ceccherini ${ }^{1}$, C. Belotti ${ }^{1}$, B. Carli ${ }^{1}$, P. Raspollini ${ }^{1}$, and M. Ridolfi ${ }^{2}$ \\ ${ }^{1}$ Istituto di Fisica Applicata "Nello Carrara" del Consiglio Nazionale delle Ricerche, Via Madonna del Piano, 10 - 50019 \\ Sesto Fiorentino, Italy \\ ${ }^{2}$ Dipartimento di Chimica Fisica e Inorganica, Università di Bologna, Viale del Risorgimento, 4 - 40136 Bologna, Italy
}

Received: 19 September 2006 - Published in Atmos. Chem. Phys. Discuss.: 18 December 2006

Revised: 8 March 2007 - Accepted: 9 March 2007 - Published: 12 March 2007

\begin{abstract}
The retrieval of concentration vertical profiles of atmospheric constituents from spectroscopic measurements is often an ill-conditioned problem and regularization methods are frequently used to improve its stability. Recently a new method, that provides a good compromise between precision and vertical resolution, was proposed to determine analytically the value of the regularization parameter. This method is applied for the first time to real measurements with its implementation in the operational retrieval code of the satellite limb-emission measurements of the MIPAS instrument and its performances are quantitatively analyzed. The adopted regularization improves the stability of the retrieval providing smooth profiles without major degradation of the vertical resolution. In the analyzed measurements the retrieval procedure provides a vertical resolution that, in the troposphere and low stratosphere, is smaller than the vertical field of view of the instrument.
\end{abstract}

\section{Introduction}

Regularization methods are frequently used to improve the conditioning of the retrieval of atmospheric constituent vertical profiles from spectroscopic measurements. The regularization is used to decrease the retrieval errors at the expenses of the vertical resolution. One regularization method effectively used in atmospheric retrievals is the Tikhonov regularization (Tikhonov, 1963) which is implemented with a constraint matrix and a parameter driving the strength of the regularization. The value of the regularization parameter controls the trade-off between precision and vertical resolution and its choice is a difficult task for which several approaches were proposed (Schimpf and Schreier, 1997; Doicu et al., 2002; Steck, 2002). Recently a new procedure to de-

Correspondence to: S. Ceccherini

(s.ceccherini@ifac.cnr.it) termine analytically the value of the regularization parameter has been proposed by Ceccherini (2005) based on the criterion that the differences between the regularized and the nonregularized profiles should be on average equal to the errors of the regularized profile. This method, requiring the nonregularized solution for the calculation of the regularization parameter, can only be applied to improve the conditioning of a problem and cannot be used to overcome ill-posed problems. A first application of this new method, named the error consistency (EC) method, is made in this paper in the case of the Level 2 analysis of the measurements of the Michelson Interferometer for Passive Atmospheric Sounding (MIPAS) (Fischer et al., 2000). MIPAS is a Fourier transform spectrometer that operates aboard the environmental satellite (ENVISAT) and measures the atmospheric limb emission in the middle infrared. The spectra measured by MIPAS contain information on the state of the atmosphere and a quantitative analysis of these spectra allows to determine several geophysical parameters of interest. The performances of this regularization are assessed in this work with some dedicated tests made using the scientific prototype, called optimized retrieval model (ORM), of the MIPAS operational level 2 code. This code performs a Gauss-Newton non-linear least square fit modified by the Levenberg-Marquardt method using a global fit strategy. It is used to retrieve from a limb-sequence of MIPAS spectra the pressure at the tangent points and the profiles of temperature and volume mixing ratio (VMR) of $\mathrm{H}_{2} \mathrm{O}, \mathrm{O}_{3}, \mathrm{HNO}_{3}, \mathrm{CH}_{4}, \mathrm{~N}_{2} \mathrm{O}$ and $\mathrm{NO}_{2}$. A detailed description of this algorithm can be found in the works of Ridolfi et al. (2000) and of Raspollini et al. (2006).

In the nominal observation mode adopted after January 2005, a MIPAS limb-scan consists of 27 spectra that look at tangent altitudes from 7 to $72 \mathrm{~km}$, with $1.5 \mathrm{~km}$ steps from 7 to $22 \mathrm{~km}, 2 \mathrm{~km}$ steps from 22 to $32 \mathrm{~km}, 3 \mathrm{~km}$ steps from 32 to $47 \mathrm{~km}, 4 \mathrm{~km}$ steps from 47 to $63 \mathrm{~km}$ and $4.5 \mathrm{~km}$ steps from 63 to $72 \mathrm{~km}$. The signal measured by the instrument is obtained with an instantaneous field of view (IFOV) equal to

Published by Copernicus GmbH on behalf of the European Geosciences Union. 
$3 \times 30 \mathrm{~km}^{2}$ (vertical height times across-track width). Since the step of the measurement grid is for some altitudes smaller than the vertical IFOV, contiguous limb scanning views have overlapping IFOVs. This situation, combined with the choice of using a retrieval grid that matches the measurement grid, determines an ill-conditioning of the inversion and the need for a regularization in order to avoid instabilities in the retrieved profiles. The ill-conditioning of the problem can be quantified by the condition number of the matrix to be inverted which for ozone is of the order of $10^{11}$. The adopted regularization is discussed in the following sections.

\section{The regularization method}

In the case of remote sensing measurements the regularization techniques are discussed in the books by Twomey (1977) and Rodgers (2000), but in order to have a consistent notation for our considerations the relevant equations are herewith recalled. The Tikhonov regularized solution of the retrieval problem can be obtained by minimizing the following cost function:

$$
\begin{aligned}
f(\boldsymbol{x}) & =(\boldsymbol{y}-\boldsymbol{F}(\boldsymbol{x}))^{T} \mathbf{S}_{\boldsymbol{y}}^{-1}(\boldsymbol{y}-\boldsymbol{F}(\boldsymbol{x}))+ \\
& +\lambda\left(\boldsymbol{x}-\boldsymbol{x}_{a}\right)^{T} \mathbf{R}\left(\boldsymbol{x}-\boldsymbol{x}_{a}\right)
\end{aligned}
$$

where $\boldsymbol{x}$ is the vector representing the profile to be determined, $\boldsymbol{y}$ is the measurement vector, $\mathbf{S}_{\boldsymbol{y}}$ is the variancecovariance matrix (VCM) describing the errors of $\boldsymbol{y}, \boldsymbol{F}(\boldsymbol{x})$ is the forward model, $\lambda$ is a positive parameter characterizing the strength of the regularization, $\boldsymbol{x}_{a}$ is an a priori estimate of the state vector and $\mathbf{R}$ is a regularization matrix. The cost function $f(\boldsymbol{x})$ contains two terms: the first term is the "chisquare" that measures how well the forward model calculated in $\boldsymbol{x}$ is able to reproduce the measurements within their errors, and the second term measures how well the retrieved profile follows some feature of $\boldsymbol{x}_{a}$ determined by the regularization matrix. In our case, where $\mathbf{R}=\mathbf{L}_{1}^{T} \mathbf{L}_{1}$ with $\mathbf{L}_{1}$ equal to the discrete first derivative operator, the vertical increments of the retrieved profile are constrained to follow those of $\boldsymbol{x}_{a}$. If $\boldsymbol{x}_{a}$ is a smooth profile, the $\mathbf{L}_{1}$ operator provides a smoothing of the retrieved profile while reducing the negative correlations between vertically adjacent values introduced by the fact that contiguous limb views have overlapping IFOVs.

The minimum of $f(x)$ may be searched iteratively using the Gauss-Newton method, that provides the following expression of $\boldsymbol{x}$ at each iteration:

$\boldsymbol{x}=\left(\mathbf{S}_{\hat{\boldsymbol{x}}}^{-1}+\lambda \mathbf{R}\right)^{-1}\left(\mathbf{S}_{\hat{\boldsymbol{x}}}^{-1} \hat{\boldsymbol{x}}+\lambda \mathbf{R} \boldsymbol{x}_{a}\right)$

where $\hat{\boldsymbol{x}}$ is the non-regularized state vector, i.e. the solution obtained when only the first term of $f(x)$ is minimized, and $\mathbf{S}_{\hat{x}}$ is its VCM. This is an unconventional expression of the solution that will be useful for the subsequent considerations and that can be reduced to the conventional expression recalling that:

$\hat{\boldsymbol{x}}=\hat{\boldsymbol{x}}_{0}+\left(\mathbf{K}^{T} \mathbf{S}_{\boldsymbol{y}}^{-1} \mathbf{K}\right)^{-1} \mathbf{K}^{T} \mathbf{S}_{\boldsymbol{y}}^{-1}\left(\boldsymbol{y}-\boldsymbol{F}\left(\hat{\boldsymbol{x}}_{0}\right)\right)$

$\mathbf{S}_{\hat{\boldsymbol{x}}}=\left(\mathbf{K}^{T} \mathbf{S}_{\boldsymbol{y}}^{-1} \mathbf{K}\right)^{-1}$

$\hat{\boldsymbol{x}}_{0}$ being the initial guess of the iteration and $\mathbf{K}$ the Jacobian matrix of $\boldsymbol{F}(\boldsymbol{x})$ calculated in $\hat{\boldsymbol{x}}_{0}$.

The solution of Eq. (2) is characterized by the following averaging kernel matrix (AKM) (Rodgers, 2000):

$\mathbf{A}_{x}=\left(\mathbf{S}_{\hat{x}}^{-1}+\lambda \mathbf{R}\right)^{-1} \mathbf{S}_{\hat{x}}^{-1}$

and by the following VCM:

$\mathbf{S}_{x}=\left(\mathbf{S}_{\hat{x}}^{-1}+\lambda \mathbf{R}\right)^{-1} \mathbf{S}_{\hat{x}}^{-1}\left(\mathbf{S}_{\hat{x}}^{-1}+\lambda \mathbf{R}\right)^{-1}$.

To determine the regularization strength the EC method (Ceccherini, 2005) can be applied. It is based on the requirement that the difference between the regularized and the nonregularized profiles weighted with the inverse of the VCM of the regularized profile must be equal to the number $n$ of points of the profile:

$(\boldsymbol{x}-\hat{\boldsymbol{x}})^{T} \mathbf{S}_{\boldsymbol{x}}^{-1}(\boldsymbol{x}-\hat{\boldsymbol{x}})=n$.

A simplified interpretation of Eq. (7) is obtained in the case that $\mathbf{S}_{\boldsymbol{x}}$ is a diagonal matrix (even if this never occurs for atmospheric profiles retrieved from remote sensing measurements). In this case the differences between the regularized and the non-regularized profiles must be on average equal to the errors of the regularized profile (measured by the square root of the diagonal elements of $\mathbf{S}_{\boldsymbol{x}}$ ).

Substituting in Eq. (7) $\boldsymbol{x}$ from Eq. (2) and $\mathbf{S}_{\boldsymbol{x}}$ from Eq. (6), with straightforward calculations the following value for $\lambda$ is obtained:

$\lambda=\sqrt{\frac{n}{\left(\boldsymbol{x}_{a}-\hat{\boldsymbol{x}}\right)^{T} \mathbf{R S}_{\hat{\boldsymbol{x}}} \mathbf{R}\left(\boldsymbol{x}_{a}-\hat{\boldsymbol{x}}\right)}}$.

In Eq. (8) the $\mathbf{S}_{\hat{x}}$, which measures the random errors due to the mapping of random radiometric noise into the retrieved profile, is used instead of the VCM of the total retrieval errors. In this way the error components due to forward model errors are neglected in the regularization process. This choice is based on the consideration that these components are usually characterized by a positive correlation between values contiguous in altitude, and therefore, the regularization does not need to account for them.

The application of this method to the MIPAS retrieval code has to take into account the following considerations. The Gauss-Newton iterative method is successful only in the case of a sufficiently weak non-linearity of the forward model. In the case of strong non-linearities some iterations of the iterative method can lead to an increase rather than to a decrease 
of the residuals. For this reason in the ORM a modification of the Gauss-Newton method, the Levenberg-Marquardt technique (Levenberg, 1944; Marquardt, 1963), is used. This latter modifies Eqs. (3) and (4) into:

$$
\begin{gathered}
\hat{\boldsymbol{x}}=\hat{\boldsymbol{x}}_{\mathbf{0}}+\left(\mathbf{K}^{T} \mathbf{S}_{\boldsymbol{y}}^{-1} \mathbf{K}+\alpha \mathbf{M}\right)^{-1} \mathbf{K}^{\mathbf{T}} \mathbf{S}_{\boldsymbol{y}}^{-1}\left(\boldsymbol{y}-\boldsymbol{F}\left(\hat{\boldsymbol{x}}_{\mathbf{0}}\right)\right) \\
\mathbf{S}_{\hat{\boldsymbol{x}}}=\left(\mathbf{K}^{T} \mathbf{S}_{\boldsymbol{y}}^{-1} \mathbf{K}+\alpha \mathbf{M}\right)^{-1} \mathbf{K}^{T} \mathbf{S}_{\boldsymbol{y}}^{-1} \mathbf{K} \\
\left(\mathbf{K}^{T} \mathbf{S}_{\boldsymbol{y}}^{-1} \mathbf{K}+\alpha \mathbf{M}\right)^{-1}
\end{gathered}
$$

where $\mathbf{M}$ is a matrix that in the ORM is diagonal, with the diagonal elements equal to those of the matrix $\mathbf{K}^{T} \mathbf{S}_{\boldsymbol{y}}^{-1} \mathbf{K}$ and $\alpha$ is a parameter that, during the retrieval iterations, is increased or decreased depending on whether the chi-square function increases or decreases while the atmospheric state is upgraded from $\hat{\boldsymbol{x}}_{0}$ to $\hat{\boldsymbol{x}}$ only when the chi-square function decreases. Accordingly, the AKM is not an identity matrix, as in the pure Gauss-Newton approach, but it is equal to:

$\mathbf{A}_{\hat{x}}=\left(\mathbf{K}^{T} \mathbf{S}_{\boldsymbol{y}}^{-1} \mathbf{K}+\alpha \mathbf{M}\right)^{-1} \mathbf{K}^{T} \mathbf{S}_{\boldsymbol{y}}^{-1} \mathbf{K}$.

Some tests on MIPAS measurements have confirmed that, because of the high non-linearity of the problem, the pure Gauss-Newton iteration often produces an increased chisquare value. The Levenberg-Marquardt method is needed to determine the minimum of the chi-square, and this need is not abated by the use of the Tikhonov regularization. For this reason we decided to use both the Levenberg-Marquardt method and the Tikhonov regularization.

Recalling that the objective of the Levenberg-Marquardt method is to reach the minimum of the chi-square and the objective of the Tikhonov regularization is to limit the oscillations of the retrieved profile, it is convenient to exploit Eq. (2) which calculates the regularized profile from the nonregularized one and to perform the two operations sequentially. First the chi-square function is minimized using the Levenberg-Marquardt method by means of the iterative application of Eq. (9), secondly, when convergence has been reached, an a-posteriori regularization defined by Eq. (2), with $\hat{\boldsymbol{x}}$ and $\mathbf{S}_{\hat{\boldsymbol{x}}}$ given by the Eqs. (9-10) at the last iteration of the minimization process, is applied. This procedure is different from the commonly used procedure that performs the regularization at each iteration step. The two procedures produce similar performances in terms of vertical resolution and retrieval errors and the adopted strategy has the advantages of lighter calculations (important for operational retrievals) and provides access to both the regularized and the non-regularized profile.

The strength of the regularization can be determined by means of the EC method using Eq. (8). The VCM of the regularized profile is given by Eq. (6) and the AKM is obtained by calculating the derivative of $\boldsymbol{x}$ (provided by Eq. 2) with respect to the atmospheric true state, taking into account that the derivative of $\hat{\boldsymbol{x}}$ with respect to the atmospheric true state is (by definition) $\mathbf{A}_{\hat{x}}$ :

$\mathbf{A}_{\boldsymbol{x}}=\left(\mathbf{S}_{\hat{\boldsymbol{x}}}^{-1}+\lambda \mathbf{R}\right)^{-1} \mathbf{S}_{\hat{\boldsymbol{x}}}^{-1} \mathbf{A}_{\hat{\boldsymbol{x}}}$

where $\mathbf{A}_{\hat{x}}$ is provided by Eq. (11) calculated at the last iteration of the minimization process.

\section{Results}

The sequential application of the Levenberg-Marquardt method and the Tikhonov regularization with EC method was applied to the MIPAS measurements acquired during the ENVISAT orbit \#17540 on 8 July 2005. The retrieval determines the profile of the considered targets and other parameters necessary to simulate the measurements (the instrumental offset and the atmospheric continuum in each of the spectral intervals used for the retrieval), however, the regularization matrix $\mathbf{R}$, which is a block-diagonal matrix, was taken different from zero only in the block that corresponds to the target profile. The a-priori vector $\boldsymbol{x}_{a}$ constraining the first derivative of the profile was chosen to be constant with all the elements equal to zero. In this way the first derivative of the profile with respect to altitude decreases with a consequent reduction of the oscillations induced by negative correlations. In order to evaluate the performances of the regularization method two retrievals were performed from the measurements of the same orbit: one without the regularization and one with it. The results of the first run, in which only the Levenberg-Marquardt method is applied, are expected to be equal to the intermediate products of the second run, which are also produced with the Levenberg-Marquardt method. However, each retrieval uses as estimate of the interfering species and as initial guess of the retrieved one the results of the previous retrievals and, since these inputs depend on whether the regularization has been applied or not, the two results are different and two independent runs of the retrieval were needed. An example of the difference between the two retrievals (represented with a line when the regularization is applied and with a line plus squares when it is not applied) is given in Fig. 1 in the case of ozone for a single limb sequence. Panel (a) shows the retrieved profiles, panel (b) shows the retrieval errors and panel (c) shows the vertical resolutions (defined as the full width at half maximum of the averaging kernel) compared with the IFOV. From Fig. 1 we can see that the regularization is able to eliminate the oscillations of the non-regularized profile with a reduction of the retrieval errors at the expenses of a degradation of the vertical resolution. The vertical resolution is only partially degraded and in the troposphere and in the low stratosphere remains smaller than the IFOV. This limited loss of resolution is an important feature, because a more drastic loss of resolution is indeed obtained when a strong regularization, such as the one obtained with the so called "L-curve" method, is adopted 


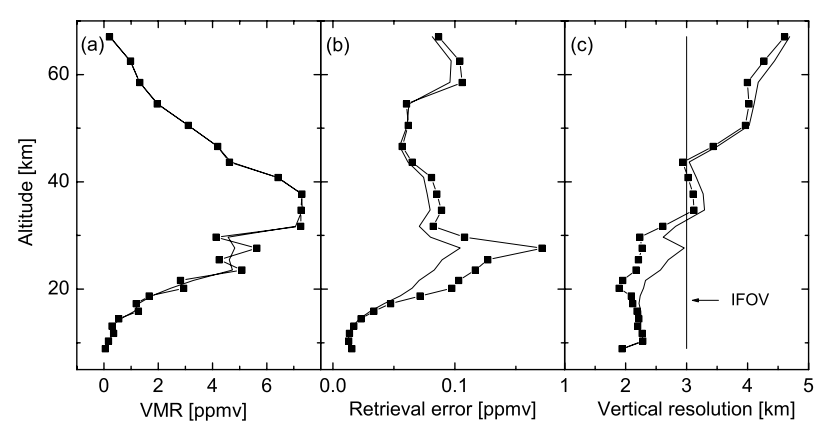

Fig. 1. Retrieved ozone profiles (a), retrieval errors (b) and vertical resolutions (c) obtained with (line) and without (line plus squares) regularization for one MIPAS limb scan.

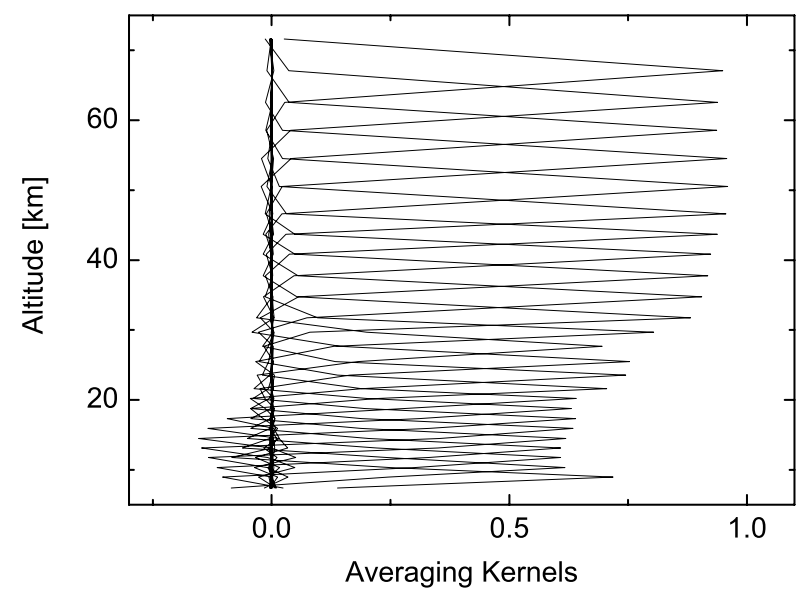

Fig. 2. Averaging kernels of the regularized profile shown in Fig. 1a.

(Ceccherini, 2005). Moreover, the regularization modifies the profile only in the altitude range where the oscillations are present, leaving unchanged the profile shape where it is already smooth. The example of Fig. 1, although referred to a single limb-scan, shows that the adopted procedure is able to provide smooth profiles with a good trade off between precision and vertical resolution. Fig. 2 shows the averaging kernels of the regularized profile reported in Fig. 1a.

In order to analyze the performances of the regularization on a significant number of retrievals the results of the ozone retrieval for all the 90 scans of the orbit have been considered. The retrieval performed using the regularization has not reached a good convergence (characterized by either a high value of $\alpha$, that determines a very poor vertical resolution, or a high value of chi-square) for 6 scans while the retrieval without the regularization has not reached a good convergence for 10 scans. This result shows that the stability of the retrieval improves when the regularization is used. In fact the a-posteriori regularization not only smoothes the retrieved profiles, but, when several species and several scans

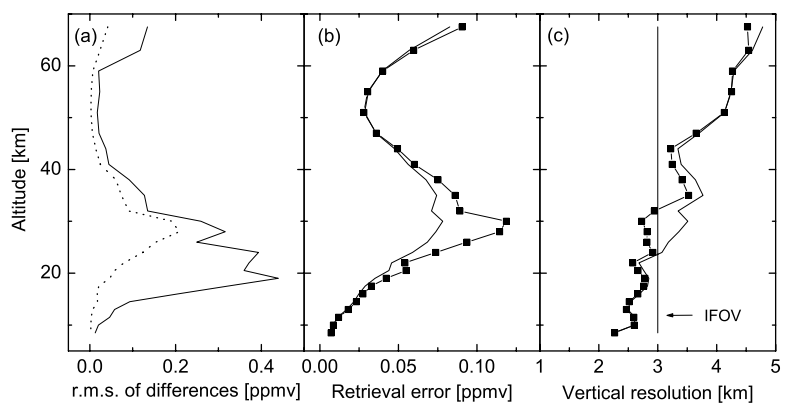

Fig. 3. Root mean square of the differences (solid line) between retrieved ozone profiles obtained with and without regularization (a) compared with the average retrieval errors (b) and the average vertical resolutions (c) obtained with (solid line) and without (solid line plus full squares) regularization for the MIPAS measurements acquired during one ENVISAT orbit. In panel (a) also the root mean square of the differences (dotted line) between the profiles $\boldsymbol{x}$ and $\hat{\boldsymbol{x}}$ of Eq. (7) is reported.

are retrieved in fall, it also contributes to stabilize the subsequent retrievals. This effect occurs through the use of more smooth and realistic profiles as assumed profiles of interfering species and as initial guess for the retrieved ones. In the following we show the results relating to the 78 scans for which a good convergence has been reached in both runs of the retrieval. In Table 1 the average values of some parameters that characterize the retrieval are reported. Among these, the $\alpha$ parameter at the end of the chi-square minimization, the number of iterations and the reduced chi-square (the chi-square normalized to the difference between the number of observations and the number of retrieved parameters) are smaller when regularization is used. This indicates that when the regularization is used the retrieval finds more easily the minimum of chi-square. Furthermore, despite the fact that the regularization for its nature of constrained retrieval should cause an increase of the chi-square, we observe a small decrease of this quantity, confirming the evolution towards more realistic profiles obtained with the regularization. The trace of the AKM measures the degrees of freedom of the retrieved vector (Rodgers, 2000; Steck, 2002) and can be calculated for either the whole state vector (i.e. including the VMR profile, the instrumental offset and the atmospheric continuum in the used spectral intervals) or be restricted to the points of the VMR profile. The traces of AKM relating to the whole state vector are nearly equal in the two cases (see Table 1). This is due to the fact that the reduction of the trace induced by the regularization is partially compensated by an increase of the trace due to a smaller value of the $\alpha$ parameter. The first effect applies mainly to the regularized parameters (retrieved VMR profile), while the second effect applies to all the retrieved parameters. This explains why when the calculation of the AKM trace is restricted to the VMR profile points a larger difference is observed between 
Table 1. Average values of parameters characterizing the ozone retrieval on 78 scans of orbit \#17540.

\begin{tabular}{|c|c|c|c|}
\hline & $\begin{array}{l}\text { Without } \\
\text { regularization }\end{array}$ & & $\begin{array}{l}\text { With } \\
\text { regularization }\end{array}$ \\
\hline Final value of the Levenberg-Marquardt parameter & 0.105 & & 0.077 \\
\hline Number of iterations & 5.28 & & 4.87 \\
\hline Reduced chi-square & 2.68 & & 2.63 \\
\hline Trace of the AKM extended to all the retrieved parameters & 48.1 & & 47.8 \\
\hline Number of the retrieved parameters & & 68.8 & \\
\hline Trace of AKM restricted to the VMR profile points & 17.7 & & 16.8 \\
\hline Number of the VMR profile points & & 23.3 & \\
\hline
\end{tabular}

the two retrievals (see Table 1). The values of the traces of the AKM have to be compared with the number of retrieved parameters and of VMR profile points. These numbers are not integers due to the fact that cloudy sweeps occasionally encountered along the orbit are excluded from the retrieval.

In Fig. 3 the root mean square of the differences between the retrieved ozone profiles obtained with and without regularization are shown by the solid line in panel (a), and, for these two profiles, the average retrieval errors and the average vertical resolutions compared with the IFOV are shown respectively in panels (b) and (c). The solid line of panel (a) and panel (b) of Fig. 3 show that the differences between the regularized and the non regularized profiles are significantly larger than the retrieval errors. The two retrievals that are compared with the solid line of panel (a) use different estimates for the interfering species and for the initial guess. So the difference shown by the solid line of panel (a) is not a measurement of the difference defined in Eq. (7). The latter difference is measured by the dotted line in panel (a) which shows the root mean square of the differences between regularized and non-regularized profiles of the same retrieval with the same estimates for the interfering species. Also the dotted line has values larger than the retrieval errors. This result is in contrast with the expectation that Eq. (7) constrains the differences between the regularized and the non-regularized profiles to be on average equal to the errors of the regularized profile. However, this expectation is based on a simplified and approximate interpretation of Eq. (7) which is not correct when the VCM $\mathbf{S}_{\boldsymbol{x}}$ is not diagonal. In practice the correlations are also important and larger changes between the regularized and the non-regularized profiles occur when correlations exist. However, in spite of this effective smoothing of the profiles the vertical resolution is only marginally degraded, see panel (c).

\section{Conclusions}

The EC method for the determination of the regularization parameter was applied for the first time to the retrieval of atmospheric parameters derived from real observations. In particular it was used for the regularization of MIPAS measurements acquired during an ENVISAT orbit and the results were compared with those obtained without regularization. The adopted regularization increases the number of retrievals for which a good convergence is reached and on average decreases the values of the final Levenberg-Marquardt parameter, of the chi-square and of the number of iterations. These features consistently point at an improved stability of the retrieval. Moreover, the regularization is able to effectively eliminate the oscillations of the non-regularized profile by modifying the profile shape only in the altitude range where the oscillations are present, leaving unchanged the shape where it is already smooth. The smoothing of the profile is obtained by reducing the retrieval errors at the expenses of a marginal degradation of the vertical resolution. In the analyzed measurements some tangent altitudes, and the corresponding retrieval grids, oversample the atmosphere with respect to the vertical IFOV. In these cases the presented retrieval procedure is able to provide smooth profiles with a vertical resolution that at these altitudes is smaller than the vertical IFOV. This is important for the analysis of the troposphere and the low stratosphere where a high vertical resolution is required.

From the practical point of view the EC method is particularly useful in operational retrievals because it ensures in a simple way the tuning of the regularization strength to the variable atmospheric conditions with the analytical calculation of a retrieval-dependent value of the regularization parameter.

Acknowledgements. This study was supported by the ESA contract 17580/03/I-OL.

Edited by: R. MacKenzie

\section{References}

Ceccherini, S.: Analytical determination of the regularization parameter in the retrieval of atmospheric vertical profiles, Opt. Lett., 30, 2554-2556, 2005. 
Doicu, A., Schreier, F., and Hess, M.: Iteratively regularized Gauss-Newton method for atmospheric remote sounding, Comput. Phys. Commun., 148, 214-226, 2002.

Fischer, H., Blom, C., Oelhaf, H., Carli, B., Carlotti, M., Delbouille, L., Ehhalt, D., Flaud, J.-M., Isaksen, I., Lopez-Puertas, M., McElroy, C. T., and Zander, R.: SP-1229 Envisat - MIPAS, ESA Publications Division, Noordwijk The Netherlands, 2000.

Levenberg, K.: A method for the solution of certain problems in least squares, Quart. Appl. Math., 2, 164-168, 1944.

Marquardt, D. W.: An algorithm for the least-squares estimation of nonlinear parameters, SIAM J. Appl. Math., 11, 431-441, 1963.

Raspollini, P., Belotti, C., Burgess, A., Carli, B., Carlotti, M., Ceccherini, S., Dinelli, B. M., Dudhia, A., Flaud, J.-M, Funke, B., Höpfner, M., Lopez-Puertas, M., Payne, V., Piccolo, C., Remedios, J. J., Ridolfi, M., and Spang, R.: MIPAS level 2 operational analysis, Atmos. Chem. Phys., 6, 5605-5630, 2006, http://www.atmos-chem-phys.net/6/5605/2006/.

Ridolfi, M., Carli, B., Carlotti, M., von Clarmann, T., Dinelli, B. M., Dudhia, A., Flaud, J.-M., Höpfner, M., Morris, P. E., Raspollini, P., Stiller, G., and Wells, R. J.: Optimized forward model and retrieval scheme for MIPAS near-real-time data processing, Appl. Opt., 39, 1323-1340, 2000.
Rodgers, C. D.: Inverse Methods for Atmospheric Sounding: Theory and Practice, Series on Atmospheric, Oceanic and Planetary Physics - Vol. 2, World Scientific, Singapore, 2000.

Schimpf, B. and Schreier, F.: Robust and efficient inversion of vertical sounding atmospheric high-resolution spectra by means of regularization, J. Geophys. Res., 102(D13), 16037-16055, 1997.

Steck, T.: Methods for determining regularization for atmospheric retrieval problems, Appl. Opt., 41, 1788-1797, 2002.

Tikhonov, A.: On the solution of incorrectly stated problems and a method of regularization, Dokl. Acad. Nauk SSSR, 151, 501$504,1963$.

Twomey, S.: Introduction to the mathematics of inversion in remote sensing and indirect measurements, Developments in Geomathematics 3, Elsevier Scientific Publishing Company, New York, 1977. 